\title{
Implementation of Envelope Analysis on a Wireless Condition Monitoring System for Bearing Fault Diagnosis
}

\author{
Guo-Jin Feng ${ }^{1} \quad$ James Gu² Dong Zhen $^{1} \quad$ Mustafa Aliwan ${ }^{1} \quad$ Feng-Shou Gu${ }^{1} \quad$ Andrew D. Ball ${ }^{1}$ \\ ${ }^{1}$ School of Computing and Engineering, University of Huddersfield, Huddersfield HD13DH, UK \\ ${ }^{2}$ School of Engineering, Manchester Metropolitan University, Manchester M156BH, UK
}

\begin{abstract}
Envelope analysis is an effective method for characterizing impulsive vibrations in wired condition monitoring (CM) systems. This paper depicts the implementation of envelope analysis on a wireless sensor node for obtaining a more convenient and reliable CM system. To maintain CM performances under the constraints of resources available in the cost effective Zigbee based wireless sensor network (WSN), a low cost cortex-M4F microcontroller is employed as the core processor to implement the envelope analysis algorithm on the sensor node. The on-chip 12 bit analog-to-digital converter (ADC) working at $10 \mathrm{kHz}$ sampling rate is adopted to acquire vibration signals measured by a wide frequency band piezoelectric accelerometer. The data processing flow inside the processor is optimized to satisfy the large memory usage in implementing fast Fourier transform (FFT) and Hilbert transform (HT). Thus, the envelope spectrum can be computed from a data frame of 2048 points to achieve a frequency resolution acceptable for identifying the characteristic frequencies of different bearing faults. Experimental evaluation results show that the embedded envelope analysis algorithm can successfully diagnose the simulated bearing faults and the data transmission throughput can be reduced by at least $95 \%$ per frame compared with that of the raw data, allowing a large number of sensor nodes to be deployed in the network for real time monitoring.
\end{abstract}

Keywords: Wireless sensor network (WSN), envelope analysis, fault diagnosis, local processing, Hilbert transformation.

\section{Introduction}

Condition monitoring $(\mathrm{CM})$ is an effective strategy to maintain the performance of modern industrial equipment. As the equipment becomes increasingly complicated, its maintenance cost has also rapidly increased. It is estimated that approximately half of operating costs can be attributed to maintenance in most processing and manufacturing operations ${ }^{[1]}$. Currently, wired online CM systems have been successfully employed in industry. However, they have been mostly restricted to large or critical machinery due to prohibitively high deployment costs. The requirement for high quality cables and their installation in harsh industrial environments contributes significantly to the system cost. In the case of wired remote condition monitoring, the installation cost may even be higher than the cost of sensors ${ }^{[2]}$. Maintaining the condition of the cables is also expensive as they often deteriorate or are damaged due to temperature fluctuation, chemical corrosions and possible incidents in a complicated working environment, which will result in less reliable $\mathrm{CM}$ performances.

Recently, wireless sensor network (WSN) is gaining popularity in CM fields because of its inherent advantages, such as ease of installation, low cost, low latency, selforganization and high reliability. WSN has already been successfully deployed for transmitting many measured sig-

Regular paper

Special Issue on Recent Advance in Automation and Computing

Manuscript received January 1, 2014; accepted September 24, 2014

Recommended by Associate Editor Yi Cao

(C) Institute of Automation, Chinese Academy of Science and Springer-Verlag Berlin Heidelberg 2015 nals, such as temperature, voltage and current ${ }^{[3,4]}$. Various standards have been developed for the wireless data transmission $^{[5]}$. Amongst these, Zigbee is a wireless protocol which is widely adopted in WSN and has been supported by industry because of its low cost, extremely low power consumption and potential to create large scale networks ${ }^{[6]}$. However, its bandwidth is limited to only 250 kilobits per second (kbps). Therefore, real-time signals with sampling rates above $250 \mathrm{kbps}$ cannot be transmitted using Zigbee systems without suffering data $\operatorname{loss}^{[6]}$. Moreover, if multiple channels or nodes coexist in the network, it will be overburdened, creating network congestion and thus packet collision.

Analysis of vibration signals have already been successfully implemented in wired CM systems ${ }^{[7]}$. However, its high bandwidth requirements limit its capabilities in WSN. Recent progressions in processing the vibration data locally ${ }^{[5]}$ (on-sensor ${ }^{[2]}$ or edge ${ }^{[8]}$ ) has been proposed to solve the bandwidth problem. Instead of directly forwarding the raw data to the sink node, the acquired data are firstly processed onboard at the sensor node and only the analyzed results are sent through the wireless network. This significantly reduces the transmission load, while all of the useful information is extracted from the data to allow for further analysis. In addition, the energy requirements for transmission of the data are a lot greater than that of data processing ${ }^{[5]}$. Therefore, local processing on the sensor node also brings the benefits of reducing power consumption ${ }^{[2,9]}$ Together with the various energy harvesting techniques ${ }^{[10]}$, the wireless condition monitoring becomes more attractive. 
Several methods have been implemented by researchers on the wireless sensor node to reduce the amount of data which needs to be transmitted through the wireless network. Common techniques include down sampling ${ }^{[8]}$, fast Fourier transformation $(\mathrm{FFT})^{[2]}$, lossless compression ${ }^{[8-9]}$ and autoregressive $(\mathrm{AR})$ coefficients $^{[9]}$. Many leading industrial companies have developed wireless CM systems embedded with local processing methods, such as CSI9420 from Emerson ${ }^{[1]}$, WiMon 100 from ABB $^{[12]}$, ADIS16229 iSensor $\AA$ wireless vibration sensor node from $\mathrm{ADI}^{[13]}$ and Echoß Wireless Vibration Monitoring System from PCB piezoelectronics $^{[14]}$.

As a classic diagnostic algorithm for extracting fault features in rotational machinery, envelope analysis has been extensively used for the early detection of faults in gearboxes and rolling element bearings ${ }^{[15,16]}$. However, its applications have been restricted to wired CM systems. Successful implementation of envelope analysis purely at the wireless sensor node for CM and fault diagnosis is rarely reported. Feng et al. ${ }^{[17]}$ took envelope analysis as an example of a local processing algorithm at the wireless sensor node and proved the efficiency of embedding local algorithms for data reduction through a wireless network. This paper provides a detailed theoretical background and implementation of the envelope analysis at the wireless sensor node. Furthermore, the performance is validated through two bearing test cases.

The remainder of this paper is organized as follows. Bearing fault mechanism and the envelope analysis algorithm are briefly introduced in Section 2. Section 3 gives the system architecture of the wireless CM system and a detailed implementation of envelope analysis. The experimental evaluation and results analysis of the proposed system are described in Section 4. Finally, Section 5 presents the conclusions.

\section{Theoretical background}

\subsection{Bearing fault mechanism}

A rolling element bearing generally consists of four parts (as shown in Fig. 1): an inner race, an outer race, rolling elements and a cage which holds the rolling elements in certain relative positions. Typically, the inner race of the bearing is mounted on a rotating shaft, and the outer race is fixed to a stationary bearing house.

Failure of a bearing can be attributed to a number of mechanisms, such as mechanical damage, crack damage, wear damage, lubricant deficiency and corrosion. Consider an example where the outer race of the bearing has a spall caused by one of the failure mechanisms. Each time the spall is rolled over, a high-level short duration force is incurred that causes the bearing to vibrate at its resonance frequency. As illustrated in Fig. 1 ( $T$ denotes the time interval between impacts) the response decays quickly due to damping ${ }^{[15]}$.

When the bearing is rotating at a steady speed, a periodical vibrating response can be captured by an accelerometer mounted on the bearing house. The frequency of this periodical response is called the fault characteristic frequency. The characteristics of this frequency depend on the faulty component, geometric dimensions and the rotational speed. The fault frequencies are varied for spalls on outer race, inner race, balls, or the cage. It is the fault characteristic frequency that is of interest in the detection of bearing faults, rather than the large amplitude responses at the high frequency resonance frequency of the bearing rings induced by the short duration impacts. For a fixed outer race bearing, its theoretical characteristic fault frequencies can be calculated using (1)-(4), and a derivation of these equations is presented in [15].

Fundamental train frequency (FTF) is defined as

$$
\mathrm{FTF}=\frac{S}{2}\left(1-\frac{B}{P} \cos \phi\right)
$$

Ball pass frequency, outer race (BPFO) is defined as

$$
\mathrm{BPFO}=\frac{N S}{2}\left(1-\frac{B}{P} \cos \phi\right) .
$$

Ball pass frequency, inner race (BPFI) is defined as

$$
\mathrm{BPFI}=\frac{N S}{2}\left(1-\frac{B}{P} \cos \phi\right) .
$$

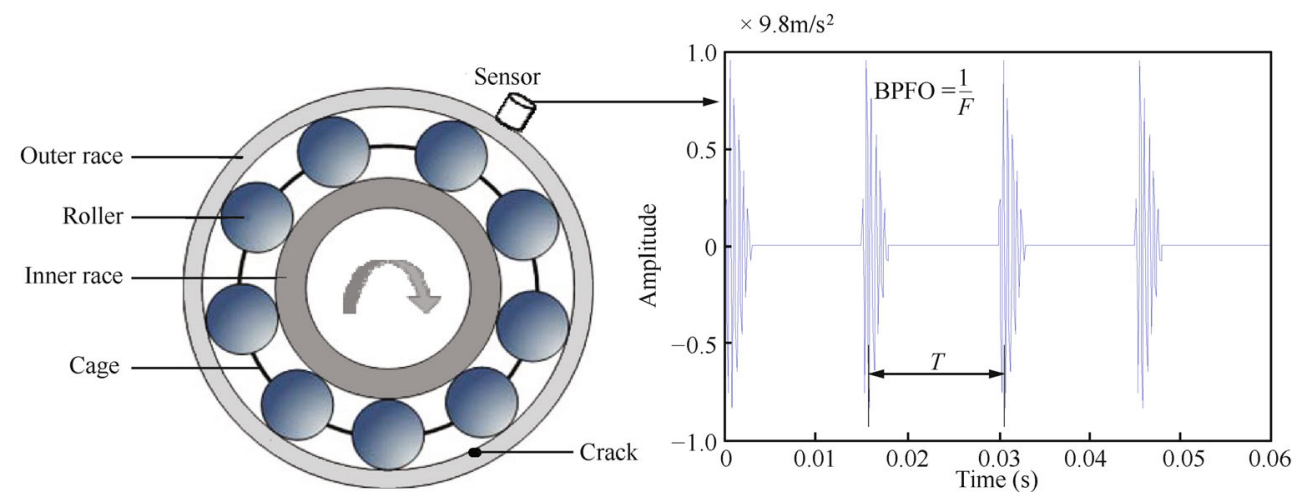

Fig. 1 Idealized vibration signature due to fault in outer race ${ }^{[15]}$ 
Ball spin frequency (BSF) is defined as

$$
\mathrm{BSF}=\frac{P S}{2 B}\left(1-\frac{B^{2}}{P^{2}} \cos ^{2} \phi\right)
$$

where $B$ is the ball diameter, $P$ is the pitch diameter, $N$ is the number of balls, $\phi$ is the contact angle and $S$ is the shaft rotation rate in Hertz. These equations are theoretical, and discrepancies arise when bearings carry significant thrust loads or if there is any slippage.

\subsection{Envelope analysis}

As described above a spall on bearing components generates a characteristic fault signal which will modulate with the bearing's resonance signal. This early stage defective signal tends to be masked by various noises such as inherent misalignments and imbalances, which makes the fault difficult to be detected by using the traditional spectrum analysis alone.

Envelope analysis has been proven as an effective method for extracting bearing fault signatures ${ }^{[16]}$, where faults have an amplitude-modulating (AM) effect on the characteristic frequencies of the machinery ${ }^{[15]}$. It has been adopted extensively for detecting fault locations in rotating machines, such as bearings, gearboxes, etc.

The procedure of implementing envelope analysis is shown in Fig. 2, where $x_{\text {in }}$ is the measured signal and $x_{\text {env }}$ is the envelope spectrum. Firstly, the measured signal is filtered using a bandpass filter which is centered in high frequency range around the system resonance. After this step, the low-frequency contents with high-amplitude caused by imbalance or misalignment are eliminated and high frequency noises from the measurement system will be suppressed. Therefore, a good signal-to-noise ratio (SNR) can be achieved ${ }^{[15]}$.

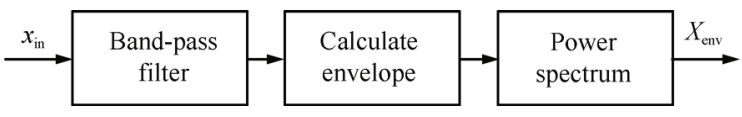

Fig. 2 Procedure of envelope analysis

Secondly, the envelope of the signal is calculated by using Hilbert transformation (HT), which is a common and effective method used to extract envelope signals ${ }^{[18]}$ and widely used in $\mathrm{CM}$ and other fields such as analyzing electrocardiogram (ECG) signals in the medical field ${ }^{[19]}$. In this method, an analytic signal $x_{\mathrm{env}}$ of the input $x_{\text {in }}$ is created using Hilbert transform, as is given in (5)-(7).

$$
\begin{aligned}
& X_{\mathrm{in}}=f f t\left(x_{\mathrm{in}}\right) \\
& X_{a}(n)= \begin{cases}X_{\mathrm{in}}(n), & \text { if } n=0, \frac{N}{2} \\
2 \times X_{\mathrm{in}}(n), & \text { if } 0<n<\frac{N}{2} \\
0, & \text { if } \frac{N}{2}<n<N\end{cases} \\
& x_{a}=\operatorname{ifft}\left(X_{a}\right)
\end{aligned}
$$

where $X_{\text {in }}$ and $X_{a}$ are the FFT of $x_{\text {in }}$ and $x_{a}$ respectively, $n$ is the index of data sequence with the length of $N$. The created analytic signal is a complex signal, where the real part is the original signal and the imaginary part is the Hilbert transform of the original signal. Thus, the envelope of the measured signal can be computed using (8).

$$
x_{\mathrm{env}}=\sqrt{x_{a} \times \operatorname{conj}\left(x_{a}\right)} .
$$

Finally, the spectrum of the envelope can be calculated using (9). Here, the mean value of the envelope $\overline{x_{\text {env }}}$ is first subtracted to remove the offset component and a Hanning window is applied on the signal to reduce spectral leakage ${ }^{[20]}$. Fig. 3 shows an evaluation of applying envelope analysis to a modulation signal, it shows that the envelope analysis method is effective in eliminating the resonance component at high frequency and can also highlight the periodical components of interest at low frequency.

$$
X_{\text {env }}=\left|f f t\left(\left(x_{\mathrm{env}}-\overline{x_{\mathrm{env}}}\right) \times \operatorname{hann}(N)\right)\right| .
$$
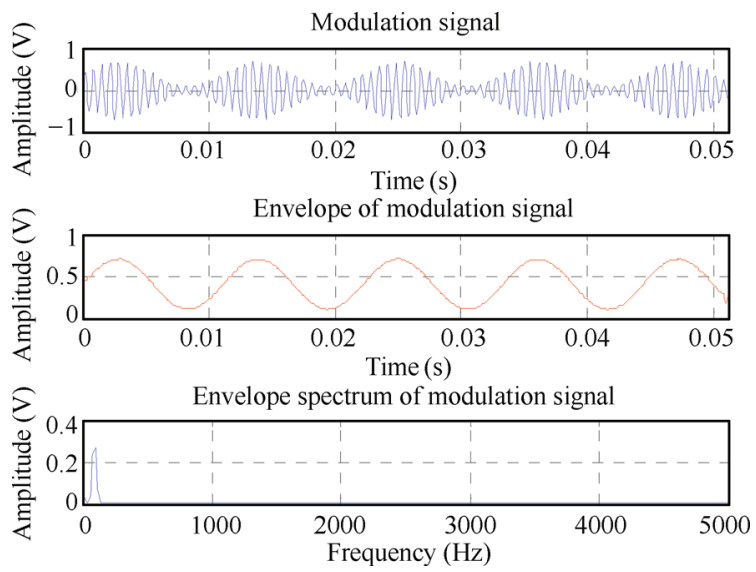

Fig. 3 Application of envelope analysis on a modulation signal

As an important part of envelope analysis HT consumes large amounts of memory and requires long computing time. By implementing the above method, the buffer for computing can be reused, which is beneficial for a processor with limited storage. In addition, the FFT implementation is the most costly part in terms of computation. In the process of implementing envelope analysis, two forward FFT in (5, 9) and one inverse FFT of (7) are computed, which has a high demand on the computing capabilities of the processor at the wireless node while in comparison the spectrum analysis only requires one forward FFT calculation.

\section{System implementation}

Wireless sensors are usually designed with limited memory size and restricted computational capabilities, as they are required to be deployed in large networks, need to be low cost, and should have low power consumption ${ }^{[5]}$. As an example, the popular wireless module Xbee Proß ZB, has only 2 kilo bytes (KB) RAM and an 8-bit processor running at $50.33 \mathrm{MHz}^{[21]}$. The system-on-chip (SOC) solution CC2530 has $8 \mathrm{~KB}$ RAM and an 8-bit processor running at $32 \mathrm{MHz}^{[22]}$. These wireless modules are not powerful enough for signal processing methods such as real time FFT 
analysis. Therefore, an additional external processor, which has much better performance yet still has low power consumption, is needed to fulfill the complex signal processing algorithms. In this paper, the Xbee pro®ZB module from Digi international ${ }^{[21]}$ is employed to set up the Zigbee network for the data transmission. This popular commercial module is effective, reliable and practical in establishing the wireless network ${ }^{[23]}$.

The overall structure of the proposed wireless CM system is shown in Fig. 4. It consists of one sink node for communicating with the host computer and several sensor nodes. The sensor node collects and processes vibration signal for the extraction of fault signatures, it then transmits these features to the sink node via a wireless Zigbee network. Finally, data from the sink node is sent to the PC via a universal serial bus (USB) connection, where it can be further analyzed to determine the condition of the plant.

The sink node has an Xbee Pro module for wireless communication and an FT232 board for communication with the host computer through a USB port. Because the feature data is relatively small and the processing speed is not demanding, the data can be received and processed in real time on the computer using Matlab.

As highlighted in the paper, the sensor node is the key component of the system. Therefore, it will be further detailed in the following three subsections with respect to three issues of signal conditioning, data processing structure and data flow of envelope analysis.

\subsection{Signal conditioning}

The vibration signal is usually collected using an accelerometer due to its low cost and good frequency response. Generally, there are two kinds of accelerometers: piezo-electric (PE) accelerometer and integrated electronics piezo-electric (IEPE) accelerometer. The PE accelerometer is more suitable for low power consumption applications since it does not need its own external power supply ${ }^{[24]}$.

Besides these two traditional accelerometers, the microelectro-mechanical systems (MEMS) accelerometer is also an option MEMS which have integrated charge amplifiers or even an analog-to-digital converter (ADC). Thus, MEMS accelerometers usually allow for a significant reduction in size, power consumption and cost compared to conventional accelerometers. Therefore, they are being used in more and more fields. For example, a digital MEMS accelerometer ADXL345, is used to detect the mechanical looseness and misalignment faults of induction motors in [25]. However, the bandwidth of most MEMS accelerometers is restricted within $2 \mathrm{kHz}$ while the fault frequencies mentioned in Section 2.1 are often in the $\mathrm{kHz}$ range ${ }^{[26]}$, which is out of the range of the MEMS sensors.

On the basis of the above considerations, a generalpurpose PE accelerometer is selected for vibration measurement. Its frequency range is from $0.5 \mathrm{~Hz}$ to $5 \mathrm{kHz}$ and the sensitivity is $16.02 \mathrm{pC} / \mathrm{ms}^{-2}$, which are the typical parameters for $\mathrm{CM}$ applications. As shown by the sensor node structure in Fig. 4, the accelerometer is connected to a charge amplifier with a gain of $3.3 \mathrm{mV} / \mathrm{pC}$ to convert the high impedance output of the accelerometer into a lower impedance one. Then, the signal is amplified by 10 times to match the dynamic range of the analog to digital converter. In addition, an anti-aliasing filter with $5 \mathrm{kHz}$ cutoff frequency is used to match up with the $10 \mathrm{kHz}$ sampling rate of the 12-bit on-chip ADC system. In particular, the charge amplifier and the anti-aliasing filter are built using the single-supply operational amplifier with rail-to-rail output so as to meet low power demands.

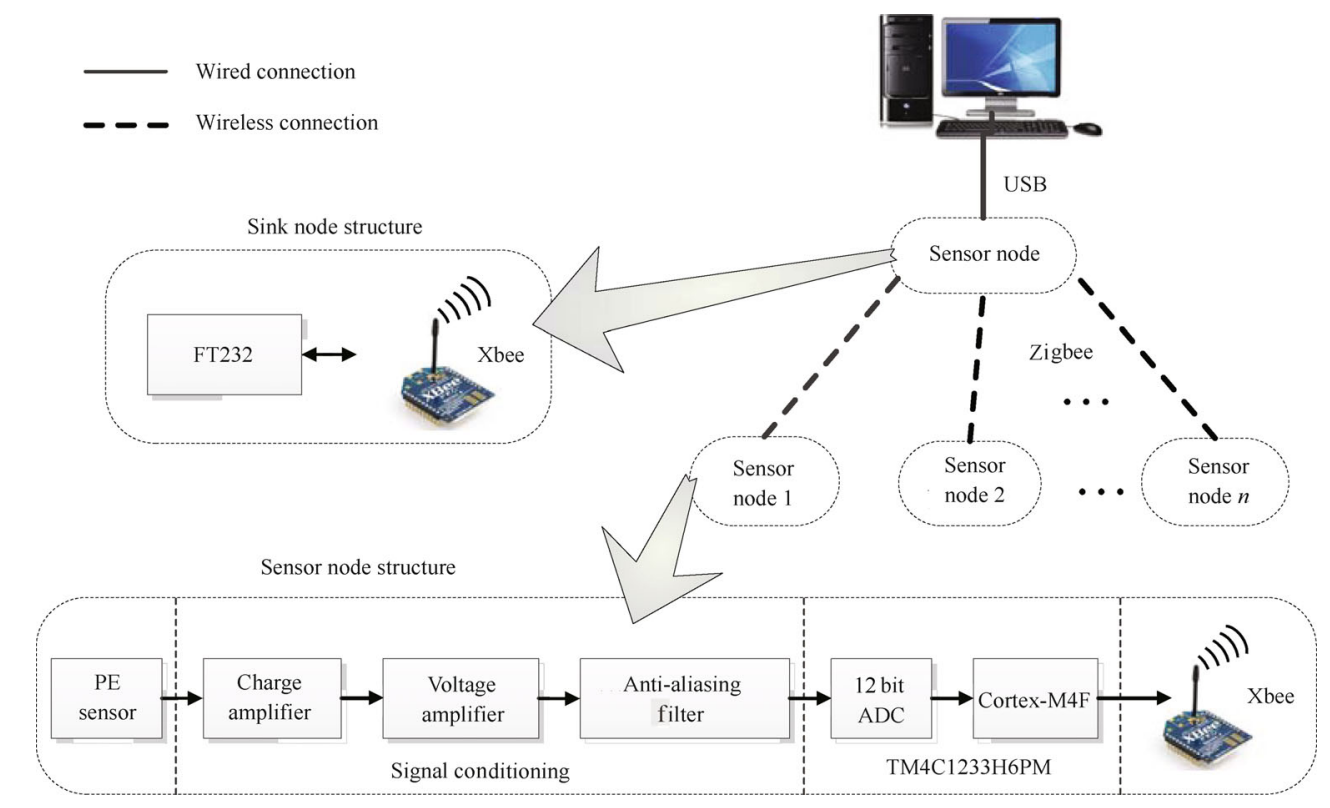

Fig. 4 Overall structure of the wireless CM system 
According to the sensor conditioning chain described above, the acceleration value $x$ can be calculated using (10).

$$
x=\frac{G_{P} \times G_{C} \times G_{F}}{2^{N}-1} \times V_{\text {ref }} \times V_{I}
$$

where $G_{P}$ is the sensitivity of the PE sensor, $G_{C}$ is gain of the charge amplifier, $G_{F}$ is the gain of the voltage amplifier, $N$ is the resolution of ADC, $V_{\text {ref }}$ is the reference voltage of $\mathrm{ADC}$, and $V_{I}$ is the conversion results of ADC.

\subsection{Data processing structure}

In this design, a low power 32-bit ARM Cortex-M4F microcontroller TM4C1233H6PM is adopted on the sensor node to process the collected vibration data. This microcontroller has $256 \mathrm{~KB}$ FLASH, $32 \mathrm{~KB}$ SRAM, two 12-bit ADCs with 1 mega samples per second (MSPS) sampling rate and abundant peripherals. Most importantly, a digital signal processing (DSP) unit and a floating point unit (FPU) are integrated inside the processor, allowing intensive computations such as FFT to be implemented ${ }^{[27]}$.

The main function of the core processor includes analog to digital conversion, implementing envelope analysis and sending processed results to the wireless module. The onchip 12-bit ADC is used for the analog to digital conversion based on cost and power consumption considerations.

The data processing structure inside the processor is illustrated in Fig. 5. Firstly, a timer, with the overflow rate set at $10 \mathrm{kHz}$, is used to trigger the analog to digital conversion. Then, after the data conversion, a direct memory access (DMA) unit, similar to a coprocessor, moves the conversion results from the ADC register to the buffers. Here, the DMA unit together with the two buffers constructs a double buffering structure, which enables the data acquisition and calculations to be completed in parallel. As a result, the signal processing can be implemented much faster $^{[28]}$.

Then, the signal is processed using the envelope analysis algorithm and the results are transmitted to the wireless module via the universal asynchronous receiver/transmitter (UART) peripheral. In this application, because data throughput of the serial port is relatively high, clear to send (CTS) and request to send (RTS) flow control methods are employed to avoid overflowing the serial buffer on the wireless module and prevent the loss of data packets. RTS and CTS flow control can be enabled using the D6 and D7 attention (AT) commands on the Xbee module ${ }^{[29]}$.

\subsection{Data flow of envelope analysis}

The data flow of envelope analysis is given in Fig. 6. The collected vibration signal is processed frame by frame, each of which is composed of 2048 points. Firstly, the data are converted from 16-bit unsigned integer to 32-bit single floating-point format for accuracy considerations. The following calculations are carried out in a large buffer with the size of $16 \mathrm{kB}$, which is twice the frame size (One data frame occupies $8 \mathrm{kB}$ memory in 32-bit format). The reason for using a double sized buffer is that FFT calculation involves a complex data operation and needs an extra buffer array for storing the imaginary part. In order to speed up the data processing, the floating point calculations are accomplished in the FPU unit.

After format conversion, the data passes through a bandpass filter to enhance the SNR, as is discussed in Section 2.2. Here, an 80-order finite impulse response (FIR) bandpass filter with $1000 \mathrm{~Hz}$ pass-band is employed. When implementing FIR filter, the data frame is divided into several smaller sub-frames with a fixed size, which then pass through the FIR filter in sequence. This allows the filtering to be accomplished using a relatively smaller buffer.

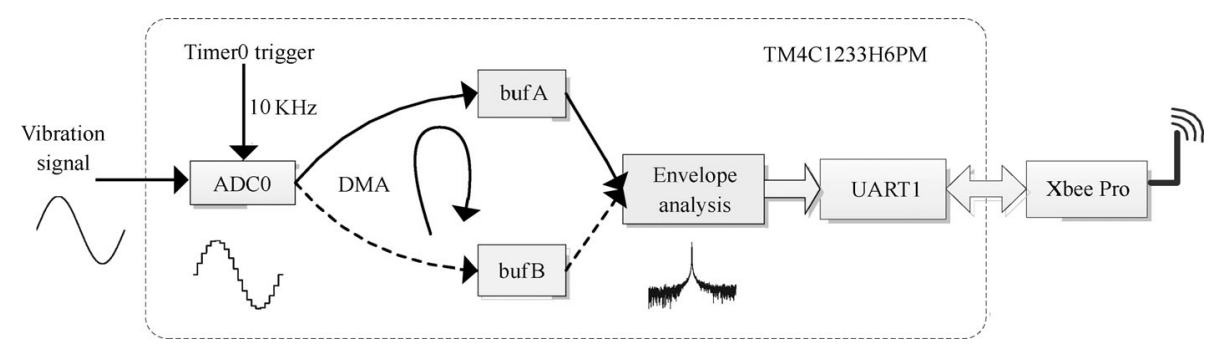

Fig. 5 Data processing flow inside the processor

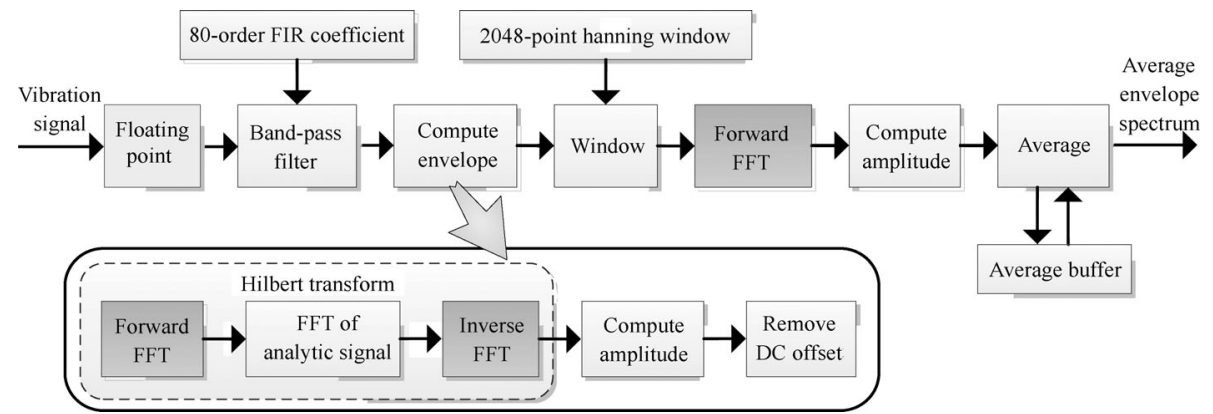

Fig. 6 Data flow of envelope analysis 
Hilbert transform is then carried out to obtain the analytic signal, which is the most costly part in terms of computation since it involves a forward FFT and an inverse FFT. After the Hilbert transform, the envelope can be acquired by simply calculating the magnitude of the analytic signal.

The next step is to compute the spectrum of the envelope signal, before which the signal is multiplied by a 2048 point Hanning window to reduce spectral leakage. Then, the envelope spectrum is obtained through a forward FFT and the amplitude calculation on the windowed signal. The fault frequencies' information should be included in the envelope spectrum. As the fault frequencies are usually in the low frequency range of several hundred Hertz, it means that only $10 \%$ of the full spectrum band needs to be transferred for a frequency range of $500 \mathrm{~Hz}$. This shows a significant data amount reduction after the processing.

At the end of the data flow, a frequency domain averaging process is added to suppress random noises and obtain a reliable envelope spectrum result. If a spectrum vector is denoted as $Y_{i}$, the averaged spectrum $\bar{Y}$ of $N$ frames of data can be obtained by

$$
\bar{Y}=\frac{1}{N} \times \sum_{i=1}^{N} Y_{i} .
$$

After the averaging process, the resultant data are converted into a 16-bit unsigned integer format and transmitted to the wireless module. It is obvious that the data size will be much smaller when only the result of averaged envelope spectrum is sent out, compared with that of sending the results of multiple envelope spectra.

In computing the envelope spectrum, the coefficients of the FIR filter and Hanning window are calculated offline and stored in the FLASH to save RAM memory. Because of the complex calculations in the implementation process, the frame buffer alone occupies $16 \mathrm{kB}$, which is half the size of the on-chip SRAM. This means 2048 points are the maximum frame length for this processor when using single floating-point numbers.

There is also a powerful DSP library for the cortex-M processors, which reduces the complexity of the code required for the signal processing. A host of algorithms such as complex arithmetic, vector operations, filter and control functions are ready to use. Because these functions have been optimized for high speed and efficiency, the development time can be obviously shortened ${ }^{[30]}$.

\section{Experimental validation}

\subsection{Experimental setup}

In order to evaluate the performance of the embedded envelope analysis algorithm on the wireless CM system, a bearing test rig was employed. As shown in Fig. 7 (a), it consists of five main parts: an electrical induction motor, shaft couplings, a DC generator, bearings and a motion shaft. Two cases were tested on two different types of bear- ings, but both with defects on their outer races. One bearing is a roller type and mounted inside the general bearing house, while the other is a ball type and placed inside the electrical motor. The induced defect of the roller bearing is shown in Fig. 7 (c) and a similar simulated fault exists on the motor bearing.

The bearing in the bearing house is a N406 cylindrical roller bearing and its geometric dimensions are listed in Table 1. During the test, the shaft ran at a full speed of $1460 \mathrm{rpm}$, i.e., $24.3 \mathrm{~Hz}$. According to Section 2.1, four defect frequencies can be calculated and the results are listed in Table 2. It can be seen that fault frequency of the simulated outer race is at $83.5 \mathrm{~Hz}$. Among the four fault frequencies, the highest one is the inner race fault frequency at $135.5 \mathrm{~Hz}$, whose $3 \mathrm{rd}$ harmonic frequency $(406.5 \mathrm{~Hz})$ is within $500 \mathrm{~Hz}$. Therefore, the band-pass filter with a bandwidth of $1000 \mathrm{~Hz}$ will include all the four fault frequencies and their 2 nd and 3rd harmonics. The bearing in the motor is a 6206 ball bearing and its characteristic frequencies are similar to those of N406.

The vibration signal was measured by the PE accelerometer, which is mounted respectively on the housing of the general bearing and on the casing of the motor horizontally.

Table 1 Specification of NSK type N406 cylindrical roller bearing

\begin{tabular}{cc}
\hline Parameter & Dimensions \\
\hline Pitch diameter $(\mathrm{P})$ & $58.979 \mathrm{~mm}$ \\
Ball diameter $(\mathrm{B})$ & $13.995 \mathrm{~mm}$ \\
Roller number $(\mathrm{N})$ & 9 \\
Contact angle $(\phi)$ & 0 \\
\hline
\end{tabular}

Table 2 Fault frequencies for bearing (N406) running at $1460 \mathrm{rpm}$

\begin{tabular}{cc}
\hline Defect location & Fault frequency $(\mathrm{Hz})$ \\
\hline Inner race (BPFI) & 135.5 \\
Outer race (BPFO) & 83.5 \\
Ball (BSF) & 48.4 \\
Cage (FTF) & 9.3 \\
\hline
\end{tabular}

\subsection{Roller bearing results}

The processing results of the roller bearing on the sensor node are illustrated in Fig. 8, which includes the raw signal, filtered signal and analyzed envelope. These middle results are captured in code composer studio (CCS) using the time graph and FFT magnitude graph tool ${ }^{[31]}$. Due to function limitations of the graph tool in CCS, the unit of $x$ axis is given in samples, which is the index of the corresponding signal array. As the sampling rate of the ADC is at $10 \mathrm{kHz}$ and the size of the FFT frame is 2048 points, the resolution of the frequency spectrum is $4.9 \mathrm{~Hz}$ per bin.

As shown in Fig. 8 (a), a DC offset exists in the raw vibration signal, and periodical spikes are observable which are caused by the defect on the outer race. From its spectrum, 


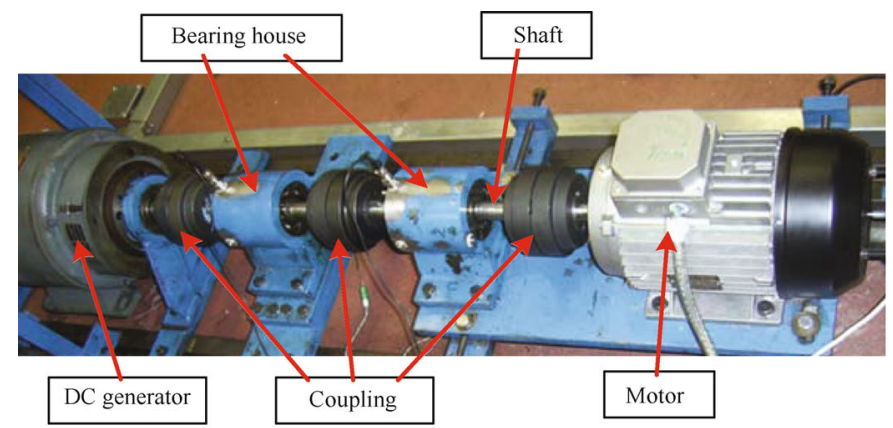

(a) Bearing test rig

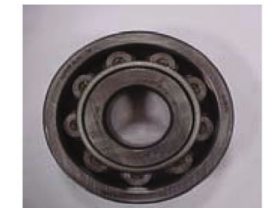

(b) Roller bearing

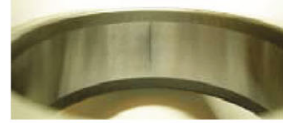

(c) Defects on outer race

Fig. 7 Test rig architecture and the roller bearing
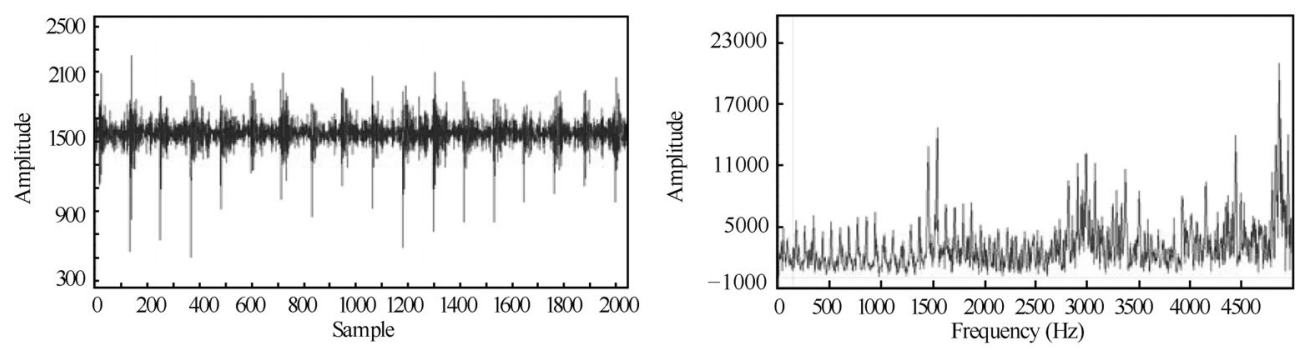

(a) Signal and spectrum of measued signal
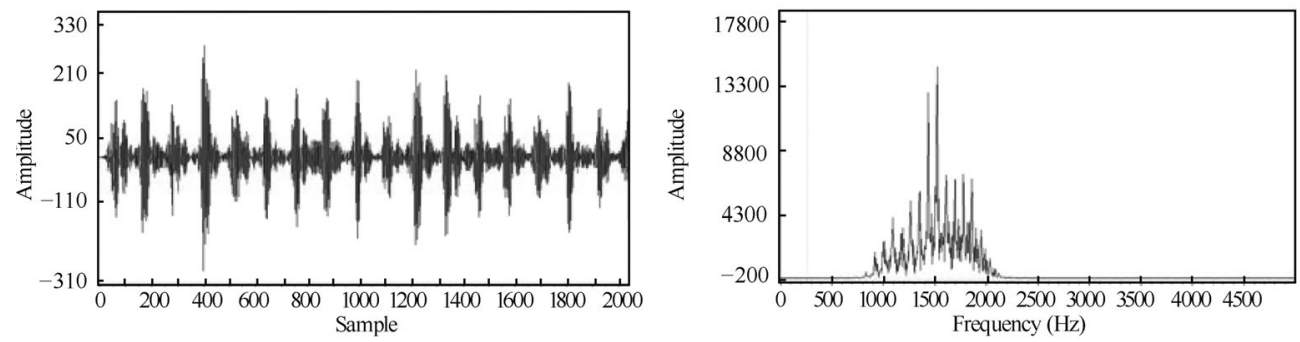

(b) Signal and spectrum of band-pass filtered signal
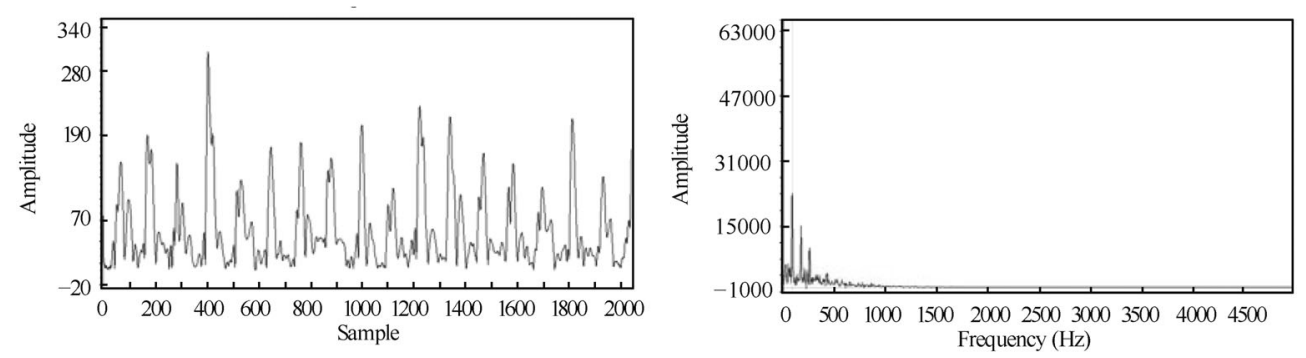

(c) Signal and spectrum of envelop signal

Fig. 8 Roller bearing

it can be seen that the signal has a wide frequency range and many discrete components, which makes it difficult to identify the fault types. Hence, the DC offset is removed from the raw signal in order to highlight the alternating current (AC) spectrum. A large frequency component appears around $1500 \mathrm{~Hz}$, which should be one of the resonance signals of the bearing. Therefore, a band-pass filter of $1 \mathrm{kHz}-2 \mathrm{kHz}$ is chosen as the band pass filter to extract this particular frequency band.

The filtered signal and its spectrum are shown in
Fig. 8 (b). The signal becomes much smoother in the time domain compared to the raw signal and only the band between $1 \mathrm{kHz}$ and $2 \mathrm{kHz}$ are kept in the frequency domain. The analyzed envelope and its spectrum are presented in Fig. 8 (c). The envelope roughly matches the outline of the filtered signal. The three low frequency components can be clearly seen as distinctive peaks in the spectrum, also frequency components above $500 \mathrm{~Hz}$ are significantly reduced.

Fig. 9 shows the envelope spectrum magnified in the low frequency range. As seen in Fig. 9, there are three distinc- 
tive spectral peaks at data sample 18,36 and 54 , whose corresponding frequencies are $87.9 \mathrm{~Hz}, 170.9 \mathrm{~Hz}$ and $258.8 \mathrm{~Hz}$ respectively. As shown in Table 2, these frequencies agree with the first three harmonics of the characteristic frequency for the outer race fault. Therefore this spectrum feature indicates the existence of an outer race fault on the roller bearing.

In addition, the spectrum in Fig. 9 is nearly flat in the frequency range higher than $500 \mathrm{~Hz}$, i.e., data sample more than 102 because of the high attenuation due to the bandpass filter. Therefore, only data in the low frequency range

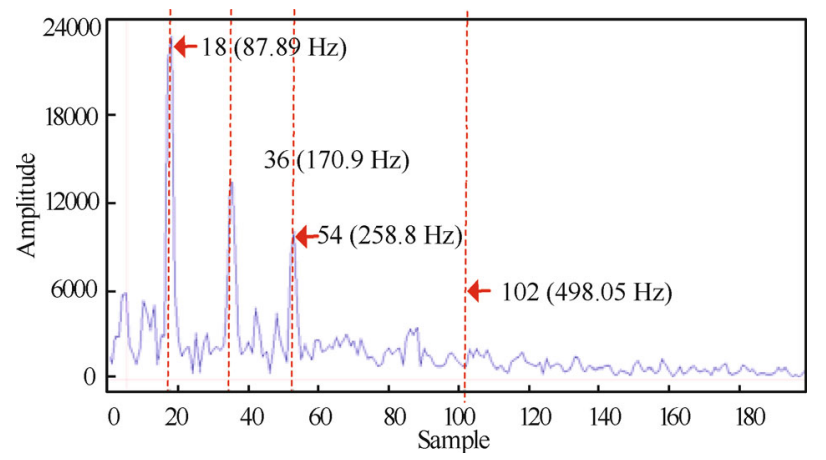

Fig. 9 Envelope spectrum magnified in low frequency range for the faulty roller bearing

(less than 103 points) need to be averaged and transmitted to the remote host computer. The amplitude of the spectrum is normalized to eliminate the effect of Hanning window and converted into the true acceleration unit according to the sensitivity given in Section 3.1. Fig. 10 presents a typical averaged envelope spectrum which is obtained after four averages on the node and transferred to the remote host machine. As it shows, the spectral peaks of interest become more distinctive and the background random components are effectively suppressed by the averaging process compared with that of Fig. 9, which allows a more reliable diagnostic result to be obtained.

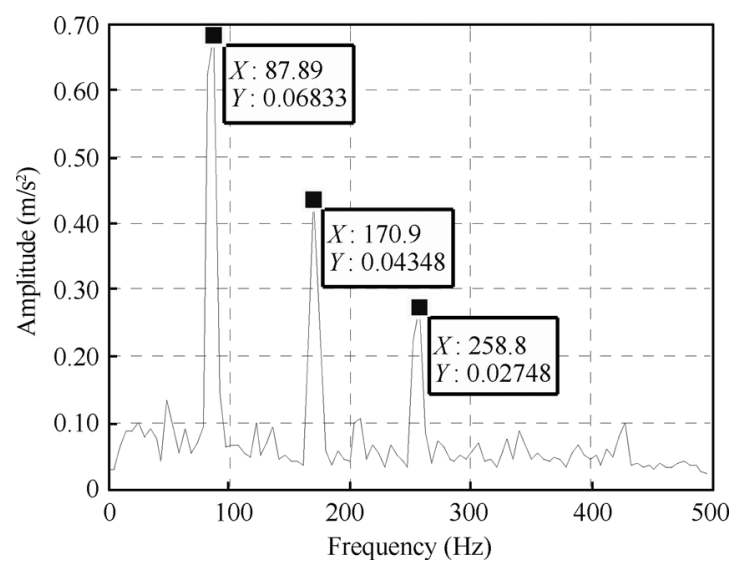

Fig. 10 Averaged envelope spectrum for the faulty roller bearing

\subsection{Motor bearing results}

To evaluate the node further, a test on an induction motor with a bearing fault was conducted. The raw signal, filtered signal and its envelope of the motor bearing vibration are shown in Fig. 11.

As given in Fig. 11 (a), periodic spikes can be also observed, but not as obvious as those in Fig. 8 (a) because the noise and other vibrations from the motor are relatively higher than those from the bearing house. From its spectrum, it can be seen that the signal also has a wide frequency range and it is difficult to determine the fault component. The spectral amplitudes around $2500 \mathrm{~Hz}$ are distinctively high. Thus, a band-pass filter with the pass band from $2 \mathrm{kHz}$ to $3 \mathrm{kHz}$ is chosen for implementing envelope analysis, which is different from that of the roller bearing because of the difference in structural resonances.

The filtered signal and its spectrum are shown in Fig. 11(b). After filtering, the signal shows more spiky characteristics due to the impact of the localized defects. The analyzed envelope and its spectrum are presented in Fig. 11 (c). Similar to the results of the roller bearing, the envelope roughly matches the outline of the filtered signal. A low frequency component has been extracted although its harmonics are not very obvious compared to those of the roller bearing.

By magnifying Fig. 11 (c) in the low frequency range, a spectral peak can be identified at $92.77 \mathrm{~Hz}$, as shown in Fig. 12. This frequency component is just one frequency bin higher than the expected $87.9 \mathrm{~Hz}$ fault frequency component. Meanwhile, it can be noticed that the frequency component at $87.9 \mathrm{~Hz}$ is quite high as well. Thus, from these features, it can be concluded the existence of the outer race fault in the motor bearing.

Using the same technique as the roller bearing test case, four averages are performed on the 103 data points in the low frequency band for the motor bearing. Then these data points are transmitted wirelessly to the remote host machine. Fig. 13 shows the averaged envelope spectrum on the remote host machine. It can be seen that the outer race fault can be clearly identified and the background noises are relatively lower than those of Fig. 12 .

\subsection{Data reduction performance}

Based on the analysis and evaluations above, the envelope analysis processing on the sensor node allows the large raw data set to be reduced into a much smaller one while the information for fault diagnosis still remains. It is this small data set that needs to be transmitted via the wireless network for online CM. In order to evaluate the performance of data reduction in different processing stages, the effective data in one frame and their equivalent data rates are given in Table 3. All the data are supposed to be transmitted with 16-bit resolution and occupy two bytes. 

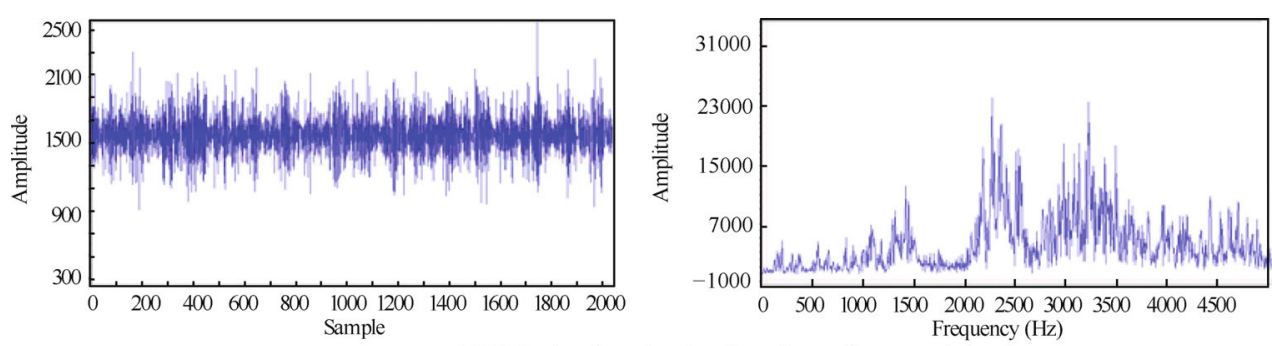

(a) Motor bearing signal and spectrum of measured signal
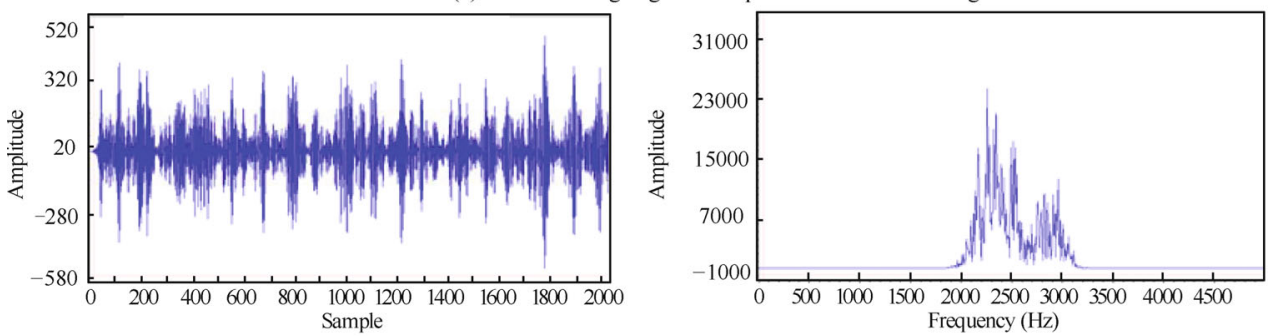

(b) Motor bearing signal and spectrum of band-pass filtered signal
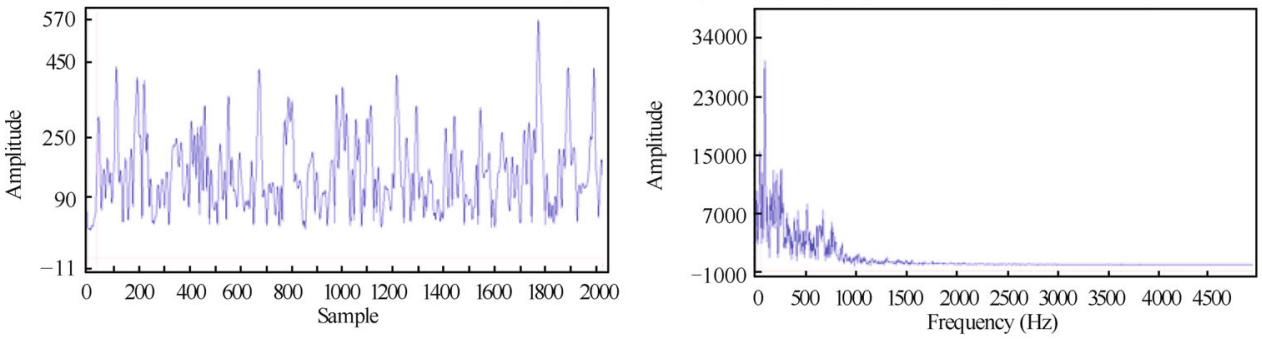

(c) Motor bearing signal and spectrum of envelope signal

Fig. 11 Motor bearing signal and spectrum

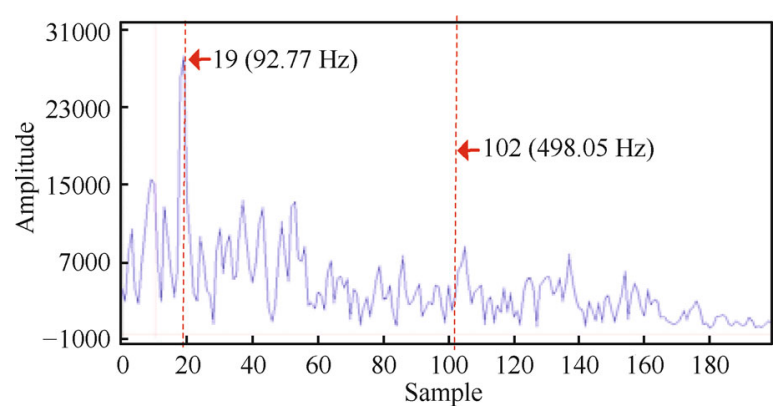

Fig. 12 Envelope spectrum magnified in low frequency range for the faulty motor bearing

Table 3 Effective data rate comparison

\begin{tabular}{ccc}
\hline Processing stage & $\begin{array}{c}\text { Data per } \\
\text { frame (bytes) }\end{array}$ & $\begin{array}{c}\text { Equivalent data } \\
\text { rate (kbps) }\end{array}$ \\
\hline Raw data & 4096 & 160 \\
Frequency spectrum & 2048 & 80 \\
Effective band data & 206 & 8 \\
in envelope spectrum & & \\
\hline
\end{tabular}

As shown in Table 3, a raw data frame of 2048 points produces a data set of 4096 bytes and its frequency spectrum requires half of that size since the spectrum is symmetric, resulting in a data reduction of $50 \%$. For the effective envelope spectrum band, only 103 points of data (206 bytes) need to be transmitted, which results in almost a $95 \%$ decrease of data size compared against the original raw data
The sampling rate in the system is set at $10 \mathrm{kHz}$. Thus, the effective data rate for the raw data is about $160 \mathrm{kbps}$. As listed in Table 3, the data rate for the effective band data is approximately $8 \mathrm{kbps}$, which is much lower than the bandwidth of the Zigbee network (250 kbps). This means that multiple such sensor nodes can be deployed in the same network.

Furthermore, if the processing results are averaged before transmitting, the data rate can be further reduced. For example, using four times the average the equivalent data rate will drop to about $2 \mathrm{kbps}$, the real-time condition monitoring would be much more efficient in that condition.

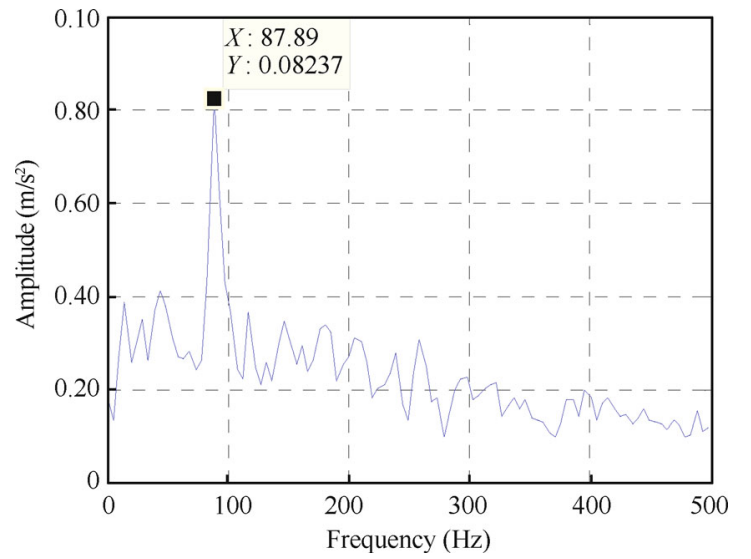

Fig. 13 Averaged envelope spectrum for the faulty motor bearing 


\section{Conclusions}

As an effective fault feature extraction algorithm, envelope analysis is implemented on a wireless sensor node for bearing fault diagnosis. A state-of-the-art and cost effective cortex-M4F core processor is employed to implement the complicated envelope analysis algorithm. The data acquisition structure, processing data flow and memory usages are optimized and the envelope analysis is achieved based on a 2048-point data frame. Using two test cases of different fault severity and signal quality, the sensor node is proved to be able to effectively identify the simulated faults. Meanwhile, the transmission data throughput can be reduced by about $95 \%$. Therefore, real-time condition monitoring based on vibration signals over the WSN can be made much more efficient. Due to the similar signal mechanisms, this wireless CM system can also be used to monitor the faults from other bearing components such as the inner race, balls and cage as well as tooth breakage of gearbox.

\section{References}

[1] C. W. de Silva. Vibration Monitoring, Testing, and Instrumentation, Boca Raton, FL, USA: CRC Press, 2007.

[2] L. Q. Hou, N. W. Bergmann. Novel industrial wireless sensor networks for machine condition monitoring and fault diagnosis. IEEE Transactions on Instrumentation and Measurement, vol.61, no. 10, pp. 2787-2798, 2012.

[3] B. Cai, X. C. Jin, S. M. Yao, J. X. Yang, X. B. Zhao, G. W. Zou. Application research on temperature WSN nodes in switchgear assemblies based on TinyOS and ZigBee. In Proceedings of the 4th International Conference on Electric Utility Deregulation and Restructuring and Power Technologies, IEEE, Weihai, China, pp. 535-538, 2011.

[4] B. Lu, L. Wu, T. G. Habetler, R. G. Harley, J. A. Gutiérrez. On the application of wireless sensor networks in condition monitoring and energy usage evaluation for electric machines. In Proceedings of the 31st Annual Conference of IEEE Industrial Electronics Society, IEEE, Raleigh, NC, USA, pp. 2674-2679, 2005.

[5] V. C. Gungor, G. P. Hancke. Industrial wireless sensor networks: Challenges, design principles, and technical approaches. IEEE Transactions on Industrial Electronics, vol. 56, no. 10, pp. 4258-4265, 2009.

[6] L. Q. Hou, N. W. Bergmann. System requirements for industrial wireless sensor networks. In Proceedings of the 2010 IEEE Conference on Emerging Technologies and Factory Automation, IEEE, Bilbao, Spain, pp. 1-8, 2010.

[7] P. J. Tavner. Review of condition monitoring of rotating electrical machines. IET Electric Power Applications, vol. 2, no. 4, pp. 215-247, 2008.

[8] L. Nachman, J. Huang, J. Shahabdeen, R. Adler, R. Kling. IMOTE2: Serious computation at the edge. In Wireless Communications and Mobile Computing Conference, IEEE, Crete Island, Greece, pp. 1118-1123, 2008.

[9] J. P. Lynch. An overview of wireless structural health monitoring for civil structures. Philosophical Transactions of The
Royal Society A: Mathematical, Physical and Engineering Sciences, vol. 365, no. 1851, pp. 345-372, 2007.

[10] J. M. Gilbert, F. Balouchi. Comparison of energy harvesting systems for wireless sensor networks. International Journal of Automation and Computing, vol. 5, no. 4, pp.334-347, 2008.

[11] CSI 9420 Wireless Vibration Transmitter-providing Accurate Vibration Monitoring in Hard-to-reach Locations, Emerson Process Management, [Online], Available: $\quad$ http://www2.emersonprocess.com/enus/brands/csitechnologies/vt/csi9420/pages/csi9420wirele ssvibrationtransmitter.aspx, August 11, 2013.

[12] Condition monitoring - Wireless vibration monitoring system, [Online], Available: http://www05.abb.com/global /scot/scot267.nsf/veritydisplay/dfad89ba8ab17a5b85257b4 10044f29d/\$file/Condition\%20Monitoring.pdf, August 16, 2013.

[13] Analog Devices' MEMS Wireless Vibration Sensing System Enables Remote Monitoring of Industrial Machine Health, [Online], Available: http://www.analog.com/ en/press-release/06_04_13_ADI_MEMS_Wireless_Vibration_ Sensing/press.html, August 16, 2013.

[14] Echo@Wireless Vibration Monitoring System, [Online], Available: https://www.imi-sensors.com/ Echo_Wireless.aspx, August 11, 2013.

[15] S. A. McInerny, Y. Dai. Basic vibration signal processing for bearing fault detection. IEEE Transactions on Education, vol. 46, no. 1, pp. 149-156, 2003.

[16] N. Tandon, A. Choudhury. A review of vibration and acoustic measurement methods for the detection of defects in rolling element bearings. Tribology International, vol. 32, no. 8, pp. 469-480, 1999.

[17] G. Feng, A. Mustafa, J. X. Gu, D. Zhen, F. Gu, A. D. Ball. The real-time implementation of envelope analysis for bearing fault diagnosis based on wireless sensor network. In Proceedings of the 19th International Conference on Automation and Computing, IEEE, London, UK, pp. 1-6, 2013.

[18] S. Holm. FFT pruning applied to time domain interpolation and peak localization. IEEE Transactions on Acoustics, Speech, and Signal Processing, vol. 35, no.12, pp. 1776$1778,1987$.

[19] L. S. Xu, Y. Wang, Y. P. Yao, C. Feng, Y. Zhao, M. Q. H. Meng. Comparison of six envelope extraction methods based on abnormal heart sounds. In Proceedings of the 2010 3rd International Conference on Biomedical Engineering and Informatics, IEEE, Yantai, China, vol. 2, pp. 813-817, 2010.

[20] F. J. Harris. On the use of windows for harmonic analysis with the discrete Fourier transform. Proceedings of the IEEE, vol. 66, no. 1, pp. 51-83, 1978.

[21] XBee®ZB-Digi International, [Online], Available: http://www.digi.com/products/wireless-wired-embeddedsolutions/zigbee-rf-modules/zigbee-mesh-module/xbee-zbmodule\#overview, March 28, 2013.

[22] Personal Area Networks - ZigBee RF4CE-CC2530-TI. com, [Online], Available: http://www.ti.com/product/cc 2530, May 29, 2013. 
[23] X. Liu, H. P. Chen, M. L. Wang, S. S. Chen. An XBeePro based energy monitoring system. In Proceedings of the 2012 Australasian Telecommunication Networks and Applications Conference, IEEE, Brisbane, QLD, Australia, pp. 16, 2012.

[24] Introduction to Piezoelectric Accelerometers, [Online], Available: http://www.pcb.com/TechSupport/Tech_Accel \#.UanZSpO1GrI, June 01, 2013.

[25] V. P. Raj, K. Natarajan, T. G. Girikumar. Induction motor fault detection and diagnosis by vibration analysis using MEMS accelerometer. In Proceedings of the 2013 International Conference on Emerging Trends in Communication, Control, Signal Processing and Computing Applications, IEEE, Bangalore, India, pp. 1-6, 2013.

[26] R. B. Randall, J. Antoni. Rolling element bearing diagnostics - A tutorial. Mechanical Systems and Signal Processing, vol. 25, no. 2, pp. 485-520, 2011.

[27] C Series for Connected MCUs-TM4C ARM CortexM4-TM4C1233H6PM-TI. com, [Online], Available: http://www.ti.com/product/tm4c1233h6pm, June 03, 2013.

[28] C. Zinner, W. Kubinger. ROS-DMA: A DMA double buffering method for embedded image processing with resource optimized slicing. In Proceedings of the 12th IEEE RealTime and Embedded Technology and Applications Symposium, IEEE, San Jose, CA, USA, pp. 361-372, 2006

[29] Digi International Inc., XBeeß/XBee-PROßZB RF Modules, [Online], Available: http://ftp1.digi.com/support/ documentation/90000976_C.pdf, May 08, 2013.

[30] TM4C ARM Cortex-M4 Microcontrollers, [Online], Available: http://www.ti.com/lsds/ti/microcontroller/tiva_ arm_cortex/c_series/tm4c_arm_cortex-m4/tools_software. page\#tivaware, June 03, 2013.

[31] Code Composer Studio (CCStudio) Integrated Development Environment (IDE) v5 - CCSTUDIO - TI Tool Folder, [Online], Available: http://www.ti.com/tool/ccstudio\# Technical Documents, December 12, 2013.

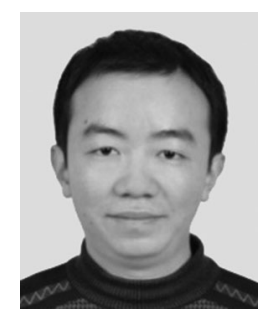

Guo-Jin Feng received the B. Sc. degree in electronic and information engineering, and M. Sc. degree in automatic devices from Shandong University of Science and Technology, China in 2009 and 2012, respectively. He is currently a Ph. D. candidate in the field of industrial machine condition motoring and fault diagnosis, University of Huddersfield, UK.

His research interests include wireless condition monitoring, real-time signal processing and wireless sensor network.

E-mail: u1273230@hud.ac.uk

ORCID iD: 0000-0001-9937-910X

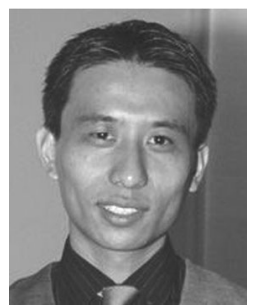

James Gu received the B. Eng. degree from University of Manchester, UK and the M. Sc. degree from Nottingham Trent University, UK. He is currently a Ph. D. candidate at Manchester Metropolitan University UK in the field of smart embedded condition monitoring.

His research interests include embedded systems, signal processing, condition monitoring and micro-electro-mechanical systems (MEMS) sensor technology.
E-mail: jx.gu@mmu.ac.uk

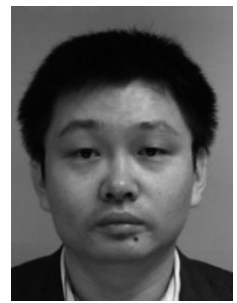

Dong Zhen received the M. Sc. degree from Shandong University of Science and Technology, China in 2009, and received $\mathrm{Ph}$. D. degree from University of Huddersfield, UK in 2012. He gained the ViceChancellor's Prize of Postgraduate Research Student of the Year in 2012 at the University of Huddersfield, UK.

His research interests include vibroacoustics analysis and machinery diagnosis, signal processing and condition monitoring.

E-mail: d.zhen@hud.ac.uk

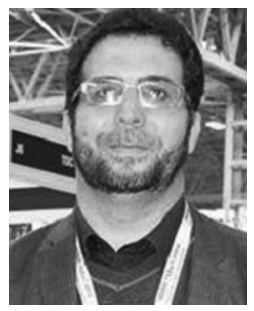

Mustafa Aliwan received the B. Sc. degree from Tajoura Academy, Libya in 1992 , and he gained a sponsorship from higher education in Libya enabling him to receive the M. Sc. degree in telecommunication and computer network scheme at the University of Salford, UK in 2009. He is currently a $\mathrm{Ph}$. D. candidate at the University of Huddersfield, UK.

His research interests include wireless sensor network and condition monitoring.

E-mail: aliwaan@yahoo.com

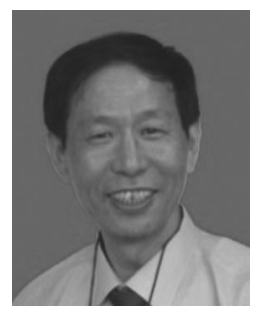

Feng-Shou Gu is an expert in the fields of vibro-acoustics analysis and machinery diagnosis, with over 20 years of research experience. He is the author of over 200 technical and professional publications in machine dynamics, signal processing, condition monitoring and related fields. $\mathrm{He}$ has experience in system modeling, various physical parameter measurements, and advanced signal processing techniques including time-frequency analysis, wavelet transforms, neural network algorithms and statistical analysis.

His research interests include vibro-acoustics related to internal combustion engines, reciprocating compressors, centrifugal pumps, electric motors, hydraulic power systems, gearboxes and bearings.

E-mail: f.gu@hud.ac.uk (Corresponding author)

ORCID iD: 0000-0003-4907-525X

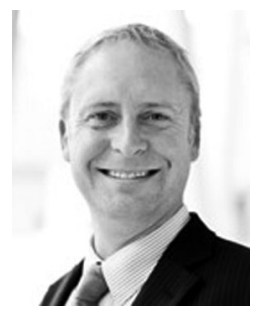

Andrew D. Ball graduated from the University of Leeds, UK with a first class honors degree in mechanical engineering, in 1987. In 1999, he was promoted to a professor of maintenance engineering, Manchester School of Engineering, UK. From 1999 to 2003 , he was the chair of the Research Committee in the Manchester School of Engineering, UK. From 2003 to 2004, he was the head of Manchester School of Engineering, UK. In 2005, he became dean of Graduate Education. In late 2007, he moved to the University of Huddersfield, UK as professor of diagnostic engineering and pro vice-chancellor for research and enterprise. He is the author of well over 200 technical and professional publications.

His research interests include machinery condition and performance monitoring, data analysis, signal processing and sensor systems design and development.

E-mail: a.ball@hud.ac.uk 